\title{
Phase Transition and Metalization of DRI According to the Quality of Iron Oxide
}

\author{
Young Min Yun ${ }^{\dagger}$ Jae Hyun Jung, Sung Kwan Seo, and Yong Sik Chu \\ Energy \& Environmental Div., Korea Institute of Ceramic Eng. \& Tech., Jinju 52851, Korea \\ (Received August 19, 2015; Revised September 2, 2015; Accepted September 3, 2015)
}

\begin{abstract}
Direct reduced iron was made using an electric furnace. The reduction ratio of direct reduced iron varied depending on the grade of iron ore. Coal played an important role as a reducing agent in making the direct reduced iron. The coal must contain a suitable amount of volatile components having high calorie values and low impurity content. In this study, oxidized pellets were directly reduced using anthracite as a reductant in an electric furnace. Direct reduction behaviors of hematite and magnetite pellets were confirmed in a coal-based experiment. Reduction behaviors were demonstrated by analyzing the chemical compositions, measuring the reducibility, and observing the phase changes and microstructure. The superior reducibility of hematite pellets can be ascribed to their high effective diffusivity, which is due to their high porosity. The quickly after reducing for 40min and achieves a high value at the end of the reduction.
\end{abstract}

Key words : Direct reduced iron, Coal based reduction, Oxidized pellets

\section{Introduction}

$\mathrm{W}$ hile the blast furnace has been used for the last 100 years to manufacture iron, Direct Reduced Iron(DRI) manufacturing process has emerged since the 1970's. The DRI manufacturing technology is a reduction method in the solid state rather than a melting method in the blast furnace, using coal, etc. as a reductant. Although this produces a smaller production quantity than the blast furnace method, it has advantages of a low initial investment cost and being able to save operation costs. ${ }^{1)}$ Owing to such advantages, the production quantity of DRI was increased from 790,000 tons in 1970 to more than 70 million tons in 2010. Recently, the DRI production quantity is approaching 80 million tons, and presumed to be increased further in the future also. ${ }^{2)}$

The direct reduced iron is manufactured by reduction of iron ores in the solid state, being manufactured by reaction between iron oxides in the irone ore and reducing gas(carbon monoxide, etc.) as shown in Equations 1, 2 and 3. Normally, the reducing gas on the iron ore surfaces permeates through fine pores to react with iron oxides. At this time, direct reduction by solid carbon and indirect reduction as gas phase-solid phase reaction by $\mathrm{CO}$ occur simultaneously, producing a $\mathrm{Fe}$ layer reduced from the surface and the part around pores. Subsequently, oxygen and reducing gas are released after being changed to gas state such as carbon dioxide, etc. ${ }^{3,4)}$

\footnotetext{
${ }^{\dagger}$ Corresponding author: Young Min Yun
}

E-mail : cementlab@kicet.re.kr

Tel : +82-55-792-2467 Fax : +82-55-792-2458

$$
\begin{aligned}
& \mathrm{Fe}_{2} \mathrm{O}_{3}+\mathrm{CO} \rightarrow 2 \mathrm{FeO}+\mathrm{CO}_{2} \\
& \mathrm{FeO}+\mathrm{CO} \rightarrow \mathrm{Fe}+\mathrm{CO}_{2} \\
& \mathrm{Fe}_{2} \mathrm{O}_{3}+3 \mathrm{C} \rightarrow 2 \mathrm{Fe}+3 \mathrm{CO}
\end{aligned}
$$

When the hematite $\left(\mathrm{Fe}_{2} \mathrm{O}_{3}\right)$ used most frequently as the raw material for iron manufacturing is reduced, it is known that hematite is transformed to magnetite $\left(\mathrm{Fe}_{3} \mathrm{O}_{4}\right)$ first, then magnetite to wustite $(\mathrm{FeO})$, after which the wustite is transformed to iron. ${ }^{5}$ ) Therefore, as shown in Fig. 1, transformation to Fe occurs first on the surfaces of iron ore particles, while reduction reaction inside particles is delayed behind that on the surface. By the above process, oxygen inside the iron ore is removed, while the reduced iron is changed to a sponge form. Thus, the direct reduced iron is also known as a Sponge Iron. ${ }^{6}$ Normally, the reduction ratio of DRI (proportion of Metal Fe in Total Fe) is in the order of $90 \%$, which may vary with manufacturing processes and raw material characteristics, etc. ${ }^{7)}$ An important factor among raw material characteristics is the iron oxide content, and the reduction ratio is characterized by being the higher, the higher the iron oxide content. Also, the shape of reduced iron may be predicted to vary with the iron oxide content. Therefore, in the present study, the reduction ratio as a function of iron ore types and iron oxide contents, etc. along with phase transformations as a function of inside structure of the reduced iron were observed.

\section{Experimental procedure}

\subsection{Starting raw material}

In the present study, imported (Australian) hematite and domestic(Jeongseon) magnetite were used for variation in 

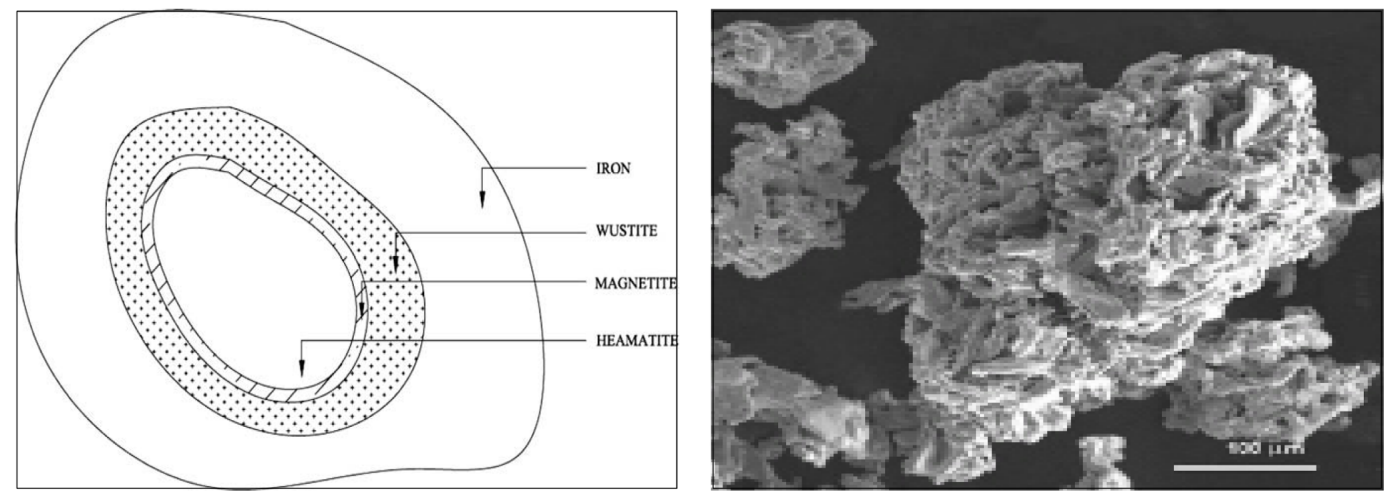

Fig. 1. Progress of reduction of solid iron ore and shape of DRI particles.

types and qualities of the iron ore. For coal, domestic anthracite(Taibaik) was used, and chemical compositions of the iron ores and the coal used in the experiment are shown in Tables 1 and 2, respectively. The major reason for using imported and domestic iron ores is to control the iron ore quality to the levels of $60 \mathrm{wt} \%, 50 \mathrm{wt} \%$ and $40 \mathrm{wt} \%$. Namely, total Fe content of hematite was $62.8 \mathrm{wt} \%$, while that of domestic magnetite- $152.5 \mathrm{wt} \%$, and that of magnetite- 2 $47.3 \mathrm{wt} \%$, etc. enabling the control for diversified qualities. The ignition loss of domestic anthracite was $70.4 \mathrm{wt} \%$ in Table 2, with the fixed carbon content being $86.6 \mathrm{wt} \%$ in Table 3. Anthracite of softening and melting properties of coal in Table 4.

\subsection{Preparation and analysis of specimens}

Each iron ore was prepared as fine powder of $150 \mu \mathrm{m}$ in

Table 1. Chemical Analyses of Iron Ore

(unit:wt\%)

\begin{tabular}{cccccc}
\hline No. & Total $\mathrm{Fe}$ & $\mathrm{Al}_{2} \mathrm{O}_{3}$ & $\mathrm{CaO}$ & $\mathrm{MgO}$ & $\mathrm{SiO}_{2}$ \\
\hline Hematite & 62.8 & 1.5 & 0.1 & 0.1 & 2.6 \\
Magnetite-1 & 52.5 & 1.8 & - & 9.3 & 10.8 \\
Magnetite-2 & 47.3 & 2.7 & 4.5 & 8.5 & 25.0 \\
\hline
\end{tabular}

Table 2. Chemical Analysis of Coal

(unit:wt\%)

\begin{tabular}{cccccccc}
\hline \multirow{2}{*}{ Anthracite } & $\mathrm{Fe}_{2} \mathrm{O}_{3}$ & $\mathrm{SiO}_{2}$ & $\mathrm{Al}_{2} \mathrm{O}_{3}$ & $\mathrm{CaO}$ & $\mathrm{MgO}$ & $\mathrm{S}^{*}$ & $\mathrm{LOI}^{* *}$ \\
\cline { 2 - 7 } & 1.41 & 13.5 & 9.0 & 0.2 & 0.3 & 0.3 & 70.4 \\
\hline
\end{tabular}

Table 3. Analysis of Coal

\begin{tabular}{ccccc}
\hline \multirow{2}{*}{ Anthracite } & $\mathrm{FC}, \mathrm{ad} / \%$ & $\mathrm{M}, \mathrm{ad} / \%$ & $\mathrm{~A}, \mathrm{ad} / \%$ & $\mathrm{~V}, \mathrm{daf} / \%$ \\
\cline { 2 - 5 } & 86.6 & 1.4 & 26.6 & 2.4 \\
\hline
\end{tabular}

-FC, ad = fixed carbon on air dry basis; M, ad=moisture on air dry basis; A, ad=ash on air dry basis; V, daf=volatile matter on dry ash free

Table 4. Softening and Melting Properties of Coal

\begin{tabular}{cccc}
\hline \multirow{2}{*}{ Anthracite } & DT & ST & HT \\
\cline { 2 - 4 } & 1,300 & 1,600 & 1,360 \\
\hline
\end{tabular}

-DT $=$ distortional temperature, $\mathrm{ST}=$ softening temperature, $\mathrm{HT}=$ half global temperature particle size, and spherical green compacts were prepared by mixing these fine powders with mixing water. At this time, the mix ratio of fine powder and mixing water was $25 \%$, while the weight of the green compact was fixed at $3 \mathrm{~g}$. The diameter of thus-produced green compact was around $10 \mathrm{~mm}$. The green compact was dried under $120^{\circ} \mathrm{C}$ condition until a constant weight was obtained. The green compact dried to a constant weight was then loaded in an alumina crucible, with the mix ratio of the green compact and the anthracite being controlled to be $1: 2$ at this time. Subsequently, reduction was carried out at $1100^{\circ} \mathrm{C}$ for $10 \mathrm{~min}$, $20 \mathrm{~min}, 40 \mathrm{~min}$ and $60 \mathrm{~min}$ after positioning the alumina crucible in an electric furnace. The exposed reduced iron was subjected to a X-ray diffraction analyzer (D5005D, Siemens Co., GERMANY) for analysis of produced crystals. The forms of the reduced iron were observed by a stereoscopic microscope, while the microstructures were observed by a scanning electron microscope. In addition, total Fe and metal $\mathrm{Fe}$ underwent a wet analysis for calculation of the reduction ratio as shown by Equation 3 .

$$
\frac{\text { Metal Fe }(w t \%)}{\text { Tatal Fe }(w t \%)} \times 100
$$

\section{Results and Discussion}

\subsection{Shape of reduced iron}

Shapes of the reduced iron(sphere) as a function of iron ore quality and reduction time were observed using a stereoscopic microscope, as shown in Fig. 2. As shown in Fig. 2, radial cracks occurred in all spheres under the reduction condition of $60 \mathrm{~min}$, and the most numerous cracks were observed in the hematite spheres. While multiple cracks were also easily observed in Hematite for the reduction time of more than $20 \mathrm{~min}$, cracks were difficult to observe in Magnetites- 1 and 2 even upon elapse of $40 \mathrm{~min}$. However, as the reduction time was increased to $60 \mathrm{~min}$, a small number of cracks were also observed in Magnetites-1 and 2. In general, the larger quantity of oxygen is present, the higher the iron oxide contents in iron ore, and this oxygen escapes by being converted to carbon dioxide in the reduction pro- 

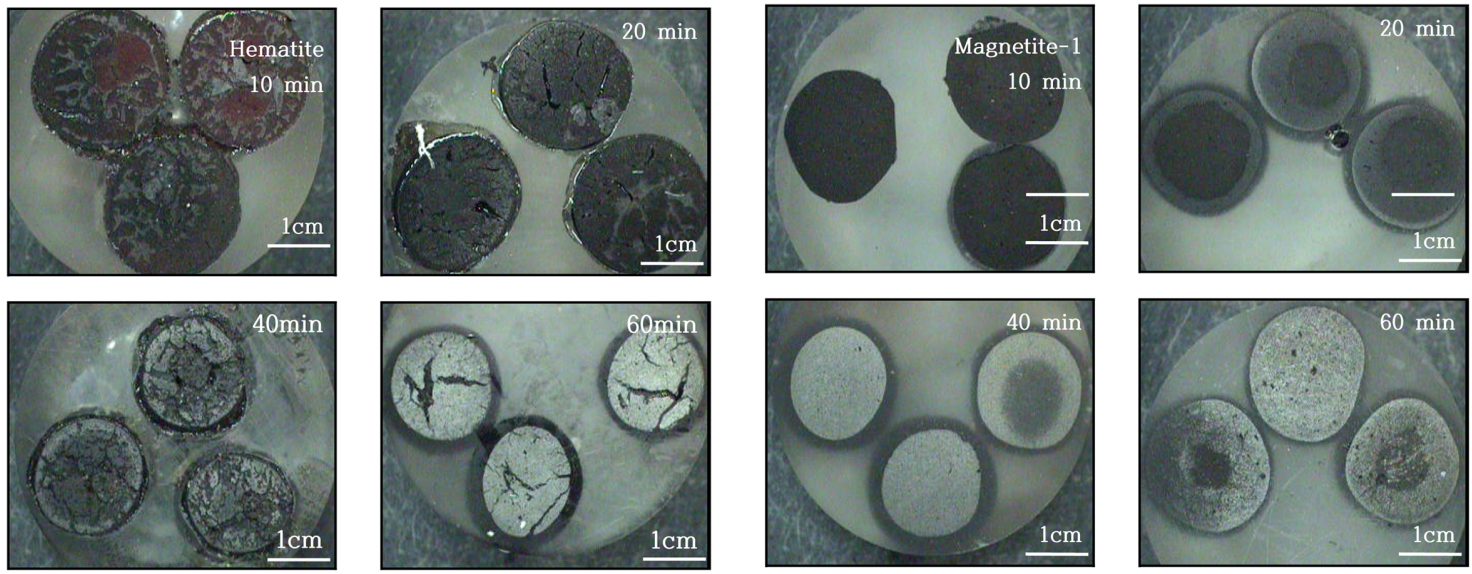

(A) Hematite DRI

(B) Magnetite-1 DRI
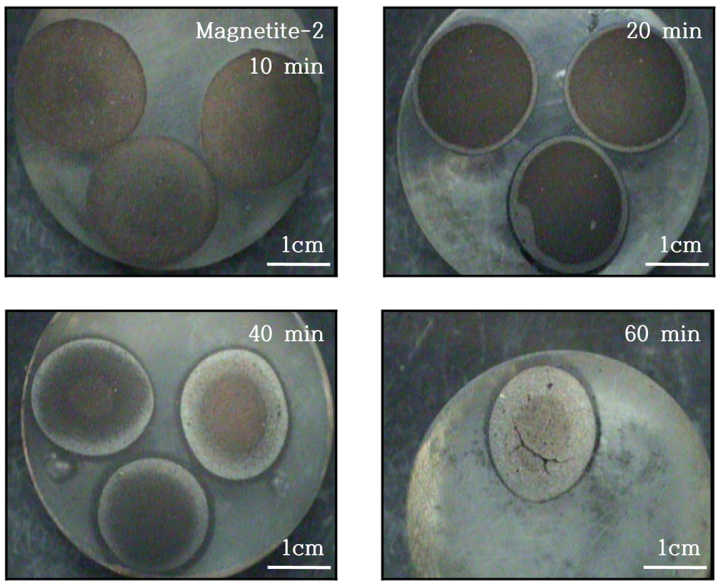

(C) Magnetite-2 DRI

Fig. 2. Morphology of DRI as a function of iron ore and reduction time.

cess. At this time, the oxygen sites in iron oxide are left as a vacant space, which was considered as having acted as the source for crack generation due to firing shrinkage. The vacant spaces also facilitate movement of reduction gas, etc. The reason that cracks of low-quality iron ore were observed after 60 min of reduction time was considered attributable to the fact that it was closely related to the content of impurities $\left(\mathrm{SiO}_{2}, \mathrm{Al}_{2} \mathrm{O}_{3}, \mathrm{MgO}\right.$, etc. $)$ as well as the content of iron oxide. Namely, the reduction ratio was decreased as a result of reduction in the space from which oxygen escaped and of obstruction of gas movement by impurities, with the decrease in reduction ratio slowing down the crack occurrence. Therefore, when the reduction time was increased to more than $60 \mathrm{~min}$, it could b expected that more cracks would be produced even in low-quality iron ores. This is a similar result to the reduction of iron ores in the rotary kiln process by coal reduction method as presented by Deqing ZHU et al. Deqing ZHU et al observed sphere cross sections while maintaining the reduction time for $5 \mathrm{~min}$ to $100 \mathrm{~min}$. Multiple cracks occurred in the reduced iron prepared by them, confirming the occurrence of radial cracks inside the reduced iron. ${ }^{8}$ )
Although changes in the sphere cross section could not be easily observed in all specimens after 10 min of reduction time, it could be confirmed that the rim part of the cross section was changed to grey color as the reduction time was increased. Particularly. in hematite, the grey part was rapidly diffused to inside, and the inside of the sphere was also completely changed to grey color under the reduction condition of $60 \mathrm{~min}$. However, the inside of magnetites- 1 and 2 was not completely changed to grey color under the reduction condition of $60 \mathrm{~min}$, and a light black area existed in the center part. This was considered to indirectly signify that the reduction rate of low-quality iron ores was so slow that the reduction reaction was not completed. Therefore, when the reduction time was increased to more than 60 min, it was presumed that even the center part of the sphere section would be changed to grey color in low-quality iron ores as well.

\subsection{Microstructure of reduced iron}

To observe microstructures of the reduced iron prepared as a function of iron ore types, a scanning electron microscope was employed, as shown in Fig. 3. The raw materials 


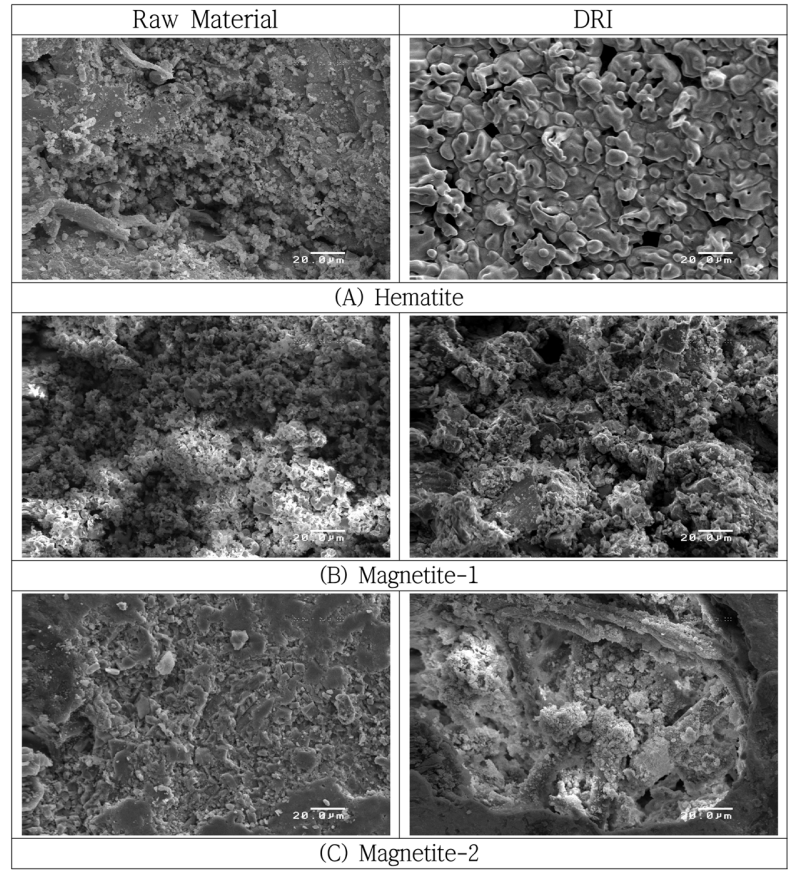

Fig. 3. Microstructures of iron ore and DRI.

in Fig. 3 correspond to hematite as a high-quality iron ore and magnetite-1, 2 as a low-quality iron ore, while the microstructures were observed by preparing DROI through respective reduction of these for $60 \mathrm{~min}$. As shown in Fig. 3, the surfaces of the reduced iron using hematite consisted of ellipsoidal particles appearing to have been dissolved, with the ellipsoidal particles being mutually connected. In addition, a small amount of fine pores were also observed. At this time, the sizes of ellipsoidal particles were $10 \sim 20 \mu \mathrm{m}$, and those of pores were less than $20 \mu \mathrm{m}$, which were also of the similar form to the microstructures of the existing reduced iron. ${ }^{9,10)}$ However, the microstructure of magnetites- 1 and 2 had a large difference from that of hematite reduced iron. In Magnetites- 1 and 2 reduced iron, most particles did not show a large difference from those in the intial raw material conditions although a small amount of ellipsoidal particles were observed. Such particle form was considered to have originated from the difference in contents of iron oxide and impurities as well as the corresponding difference in reduction ratios.

\subsection{Crystallinity analysis of reduced iron}

For crystallinity analysis of the reduced iron produced as a function of iron ore qualities, an X-ray diffraction analyzer was employed, and each X-ray pattern is shown in Fig. 4. Major crystal phase of hematite iron ore before firing was $\mathrm{Fe}_{2} \mathrm{O}_{3}$, while that of magnetites- 1 and 2 was $\mathrm{Fe}_{3} \mathrm{O}_{4}$. Under the reduction time condition of $20 \mathrm{~min}, \mathrm{Fe}_{2} \mathrm{O}_{3}$ peaks disappeared from all of hematite and magnetites-1, 2 while $\mathrm{Fe}_{3} \mathrm{O}_{4}$ peaks remained. $\mathrm{FeO}$ peaks were produced under the condi-

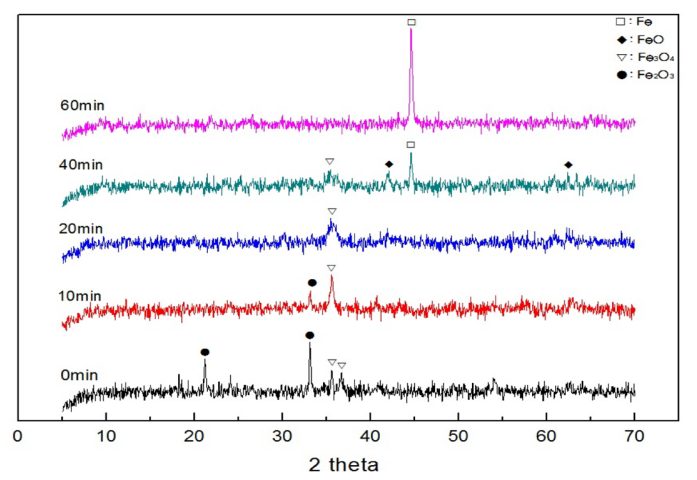

(a) Hematite

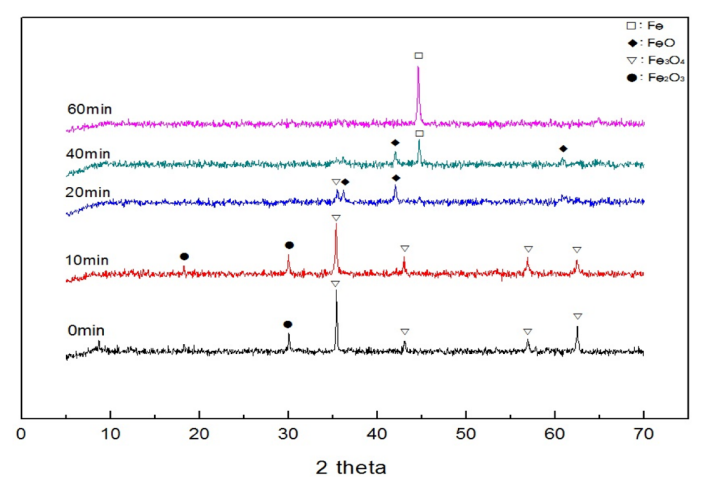

(b) Magnetite-1

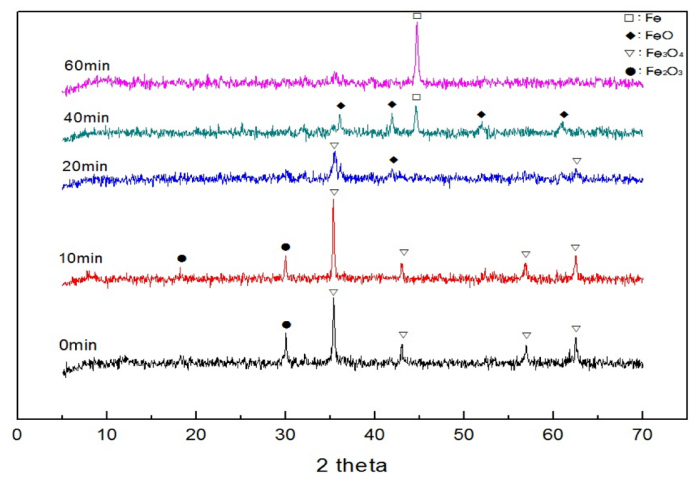

(c) Magnetite-2

Fig. 4. XRD patterns of DRI. 
tion of $40 \mathrm{~min}$, and particularly, only $\mathrm{Fe}$ peaks were observed under the reduction condition of $60 \mathrm{~min}$ so that most of the reduction could be confirmed to have been completed. Namely, as generally known, the change in crystal phases in the reduction process of iron ores occurred in the order of $\mathrm{Fe}_{2} \mathrm{O}_{3} \rightarrow \mathrm{Fe}_{3} \mathrm{O}_{4} \rightarrow \mathrm{FeO} \rightarrow \mathrm{Fe}$ for hematite, and in the order of $\mathrm{Fe}_{3} \mathrm{O}_{4} \rightarrow \mathrm{FeO} \rightarrow$ Fe for magnetite. ${ }^{11)}$ For production of crystal phases in magnetite as a function of iron ore qualities per reduction time, no large differences were observed.

\subsection{Reduction ratio of reduced iron}

After reduction of Hematite and magnetite-1, 2 for $60 \mathrm{~min}$, Total Fe and Metal Fe were analyzed, with the corresponding calculated reduction ratios being shown in Table 5. Contents of Total Fe were $86.9 \%, 62.2 \%$ and $49.1 \%$,respectively, while those of Metal $\mathrm{Fe}$ were $80.5 \%, 54.7 \%$ and $41.2 \%$, respectively. Namely, it could be confirmed that the contents of Total Fe and Metal Fe were also changed with the same tendency after reduction as a function of Total Fe contents of the initial iron ore. Reduction ratios were $92.6 \%$, $88.0 \%$ and $83.9 \%$, respectively, showing the most excellent reduction ratio for the imported hematite. As mentioned above, this is considered attributable to the fact that movement of carbon monoxide and carbon dioxide in high-quality iron ore was easier so as to contribute to the increase in reduction ratio. Exchange and diffusion of gaseous substances such as $\mathrm{CO}$ or $\mathrm{CO}_{2}$ and solid substances such as $\mathrm{Fe}$ atom, C, etc. occur through pores or lattice defects of the reduced $\mathrm{Fe}$ layer. In general, fine pores were produced inside the iron ore upon removal of oxygen from inside of the iron ore, i.e., as iron oxides were changed to iron. Fine pores facilitated movement of carbon monoxide into the iron ore, simultaneously performing the role as a discharge path for carbon dioxide as well. However, a relatively smaller amount of fine pores were produced in low-quality iron ores with a large amount of impurities such as $\mathrm{SiO}_{2}, \mathrm{Al}_{2} \mathrm{O}_{3}$ and $\mathrm{MgO}$, etc., so that the final reduction ratios must have been low. Such feature is the result which may also be indirectly confirmed in Figs. 1 and 2.

Figure 5 is a graph for the reduction ratio as a function of reduction time per iron ore type, showing a tendency that the reduction ratio was increased as the reduction time was increased. While almost no reduction occurred under the reduction conditions of $10 \mathrm{~min}$ and $20 \mathrm{~min}$, the result was obtained where the reduction ratio was rapidly increased upon elapse of $40 \mathrm{~min}$ in the reduction time. Although no large differences were exhibited up to $20 \mathrm{~min}$ in the reduction ratio as a function of iron ore types, a difference in the reduction ratios began to be shown under the condition of 40 min. At this time, the reduction ratio of hematite was $30 \%$, while that of magnetite- 2 was $16 \%$ with the difference in reduction ratios being $14 \%$. However, as the reduction time was increased to $60 \mathrm{~min}$, the difference in reduction ratios between hematite and magnetite- 2 showed a tendency of being narrowed down to about $9 \%$. This was considered to
Table 5. Chemical Analysis of Direct Reduction Iron

\begin{tabular}{cccc}
\hline \multirow{2}{*}{ Raw Material } & \multicolumn{2}{c}{ Chemical Analysis (wt\%) } & $\begin{array}{c}\text { Metalization } \\
\text { degree (\%) }\end{array}$ \\
\cline { 2 - 3 } & Total Fe & Metal Fe & 80.5 \\
\hline Hematite & 86.9 & 54.7 & 82.6 \\
Magnetite-1 & 62.2 & 41.2 & 83.0 \\
Magnetite-2 & 49.1 & & 83.9 \\
\hline
\end{tabular}

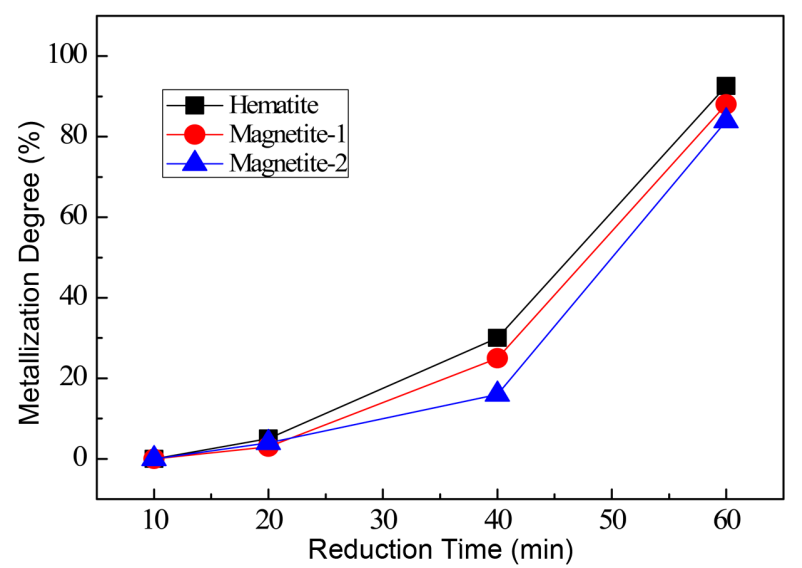

Fig. 5. Metalization degree vs reduction time.

be the result allowing confirmation of the fact that the reaction rate of hematite was somewhat faster while that of magnetite- 2 was slow at the early stage of reduction reaction. Also, it was considered to mean that low-quality iron ores with low $\mathrm{Fe}$ contents could also participate in the reduction reaction when a sufficient time for reduction reaction was secured.

\section{Conclusions}

In the present study, crystal phases and changes in reduction ratio, etc. of direct reduced iron were observed as a function of iron ore quality, and the following conclusions were derived.

1) For a sphere cross section of the reduced iron, there was a difference in crack status as a function of iron ore quality, and particularly, multiple cracks existed inside hematite iron ores. This was considered to indicate that lattice defects (oxygen vacancies inside iron oxide) acted as the source for crack occurrence.

2) It could be confirmed that Hematite was changed to the reduced iron after initial change from $\mathrm{Fe}_{2} \mathrm{O}_{3}$ to $\mathrm{Fe}_{3} \mathrm{O}_{4}$ and $\mathrm{FeO}$, and magnetite after initial change from $\mathrm{Fe}_{3} \mathrm{O}_{4}$ to $\mathrm{FeO}$. At this time, while the reduced iron using hematite could be confirmed to consist of particles in a round form, the reduced iron using magnetite could be seen to have a relatively smaller amount of particles in a round form.

3) As the reduction ratio showed a close correlation with the iron ore quality, the highest reduction ratio (92.6\%) was expressed in the hematite iron ore with the iron oxide content of $62.8 \%$. Namely, the reduction ratio showed an 
increasing tendency as the iron oxide content was increased (or the impurity content was decreased). This was considered attributable to the fact that gas movement through attice defects and cracks inside the reduced iron was facilitated.

\section{REFERENCES}

1. M. Kirschen, K. Badr, and H. Pfeifer, "Influence of Direct Reduced Iron on the Energy Balance of the Electric Arc Furnace in Steel Industry,” Energy, 36 [10] 6146-55 (2011).

2. 2011 World direct reduction statistics, www.midrex.com, Audited by WORLD STEEL.

3. Y. MAN, J. Feng, Y. Chen, and J. Zhou, "Mass Loss and Direct Reduction Characteristics of Iron Ore-coal Composite Pellets," J. Iron Steel Res. Int., 21 [12] 1090-94 (2014).

4. T. Zhang, C. Lei, and Q. Zhu, "Reduction of Fine Iron Ore via a Two-step Fluidized Bed Direct Reduction Process," Powder Technol., 254 1-11 (2014).

5. N. R. Dey, A. K. Prasad, and S. K. Singh, "Energy Survey of the Coal Based Sponge Iron Industry," Case Studies in
Thermal Engineering, 6 1-15 (2015).

6. D. Mohanty, A. Chandra, and N. Chakraborti, "Genetic Algorithms Based Multi-objective Optimization of an Iron Making Rotary Kiln," Comput. Mater. Sci., 45 [1] 181-88 (2009).

7. Y. Guo, J. Gao, H. Xu, K. Zhao, and X. Shi, "Nuggets Production by Direct Reduction of High Iron Red Mud," J. Iron Steel Res. Int., 20 [5] 24-7 (2013).

8. D. Zhu, V. Mendes, T. Chun, J. Pan, Q. Li, J. Li, and G. Qiu, "Direct Reduction Behaviors of Composite Binder Magnetite Pellets in Coal-based Grate-rotary Kiln Process," ISIJ Int., 51 [2] 214-19 (2011).

9. D. Guo, M. Hu, C. Pu, B. Xiao, Z. Hu, S. Liu, X. Wang, and $\mathrm{X}$. Zhu, "Kinetics and Mechanisms of Direct Reduction of Iron Ore-biomass Composite Pellets with Hydrogen Gas," Int. J. Hydrogen Energy, 40 [14] 4733-40 (2015).

10. R. Sen, S. Dehiya, U. Pandel, and M. K. Banerjee, "Utilization of Low Quality Coal for Direct Reduction of Mill Scale to Obtain Sponge Iron: Effect of Reduction Time and Particle Size," Procedia Earth and Planet. Sci., 11 8-14 (2015).

11. OSIL Process, Indian Patent No.:16457. 